\title{
The role of disordered angiogenesis tissue markers (sFlt-1, PIGF) in present day diagnosis of preeclampsia
}

\author{
Sebastian Kwiatkowski ${ }^{1}$, Ewa Kwiatkowska ${ }^{2}$, Andrzej Torbe ${ }^{1}$ \\ 'Clinical Department of Obstetrics and Gynecology, Pomeranian Medical University in Szczecin, Poland \\ ${ }^{2}$ Clinical Department of Internal Medicine, Nephrology and Transplantation, Pomeranian Medical University in Szczecin, Poland
}

\begin{abstract}
Preeclampsia and conditions associated with impaired placental perfusion develop in almost $10 \%$ of all pregnancies. Pathologic angiogenesis is one of the processes observed in preeclampsia. sFIt-1, PIGF and the sFIt-1/PIGF ratio are new and promising angiogenesis-related biomarkers. Our paper describes the present status of, and clinical practice opportunities for, these factors.

According to present data, sFlt-1, PIGF and the sFIt-1/PIGF ratio are very useful tools in assessing placental angiogenesis abnormalities associated with preeclampsia and can be use in clinical practice.
\end{abstract}

Key words: sFIt-1; PIGF; preeclampsia

Ginekologia Polska 2019; 90, 3: 173-176

\section{INTRODUCTION}

Preeclampsia and conditions associated with impaired placental perfusion develop in almost $10 \%$ of all pregnancies [1]. The last two decades have seen a $25 \%$ rise in the prevalence of these pathologies in the United States [2]. According to the statistical data, clinical syndromes developing secondarily to placental ischemia account for almost $50 \%$ of iatrogenic premature births, $50 \%$ of hospitalizations in pathological pregnancy departments and $25 \%$ of intensive care stays among pregnant patients [3]. Every year, preeclampsia causes nearly 60 thousand deaths among pregnant women [4]. Presently, we are unable to successfully treat these conditions, and the only effective therapy available is termination of the pregnancy. However, recent years have witnessed the emergence of diagnostic methods that have considerably improved our ability to predict pathological symptoms and thus the quality of our monitoring of women and fetuses.

Currently, literary sources concerned with this topic distinguish between early- and late-onset forms of preeclampsia [5]. The early-onset form develops prior to 32-34 weeks of gestation and is definitively connected with impaired trophoblast invasion. The insufficient transformation of the spiral arteries leads to ischemia, uteroplacental compartment hypoxia, and subsequently, to pathological pro- cesses such as elevated oxidative stress, inflammation or angiogenesis disorders arising. As a consequence of this, generalized endothelial damage occurs and various clinical symptoms develop, such as arterial hypertension, proteinuria, increased liver damage marker levels, eclampsia and intrauterine growth restriction [6]. The late-onset form of preeclampsia, which has a similar clinical presentation, develops after 34 weeks' of gestation and, according to the current data, there is only a slightly inadequate transformation of the spiral arteries, and the main role is played by maternal factors, e.g. metabolic syndrome [7]. It is also possible that a contributing factor for the development of late-onset preeclampsia syndromes is patient-specific programmed placental senescence.

\section{CURRENT STATUS}

Formerly, preeclampsia diagnosis was mainly based on recognizing clinical symptoms and studying the analytical parameters of laboratory tests that revealed the degree of organ damage. However, these analytical criteria were frequently so ambiguous that making the right diagnosis was difficult and required a process of elimination, which took a substantial amount of time. A consequence of the approach was a marked delay in diagnosis and, frequently, 
very serious consequences for the mother and her child. Presently, we are aiming to work out a more precise diagnostic process which is capable of exploring the earlier stages of the condition's development. Determining the presence of angiogenesis disorder-related markers in the uteroplacental compartment is one method to achieve that objective. Already, fms-like tyrosine kinase (sFlt-1 — soluble tyrosine kinase receptor) and placental growth factor (PIGF) laboratory kits have been marketed. sFlt-1 is produced by the syncytiotrophoblast, and its concentrations are only a few times the concentrations of PIGF in physiological pregnancies [8]. In hypoxia, or impaired perfusion of the placenta, the trophoblast produces massive amounts of sFlt1 , and the concentrations recorded in maternal circulation are at least several dozen times the levels of PIGF [9]. Such elevated levels of sFlt-1 have a dysfunctional effect that are responsible for most of the clinical and laboratory symptoms observed in preeclampsia [10].

PIGF has a stabilizing impact on endothelial function. It exerts a positive effect on, for example, placental function, but as sFlt-1 damage to the trophoblastic structures increases, its concentration is gradually reduced. Moreover, sFlt-1 is responsible for neutralizing PIGF and restricting its availability [11]. The situation observed under homeostatic conditions in normal pregnancies on the one hand, and in angiogenesis disorders accompanying preeclampsia on the other, is best represented by the diagram below (Fig. 1).

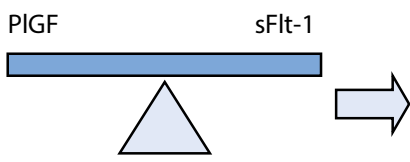

Normal pregnancies

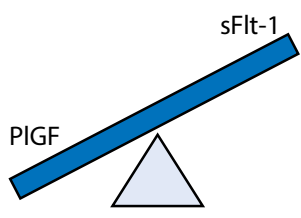

Preeclampsia
Figure 1. Diagram of the balance of angiogenesis markers in normal pregnancies and their imbalance in preeclampsia [12]

Changes in the sFlt-1 and PIGF concentrations precede the appearance of clinical symptoms and the biochemical parameters of preeclamptic organ damage by approximately 5 to 6 weeks [13].

In diagnosing preeclampsia, PIGF can be used as early as the first trimester. Combining biophysical examinations with biochemical marker assessments has enabled the discovery of an optimal model for detecting those patients with a higher risk of developing early-onset preeclampsia. This risk assessment algorithm uses the pregnant patient's medical history data, mean arterial pressure (measured twice on both the arms - MAP), uterine arterial pulsatility index, and PAPP-A and PIGF concentrations [14].
The model was modified in 2016 by proposing that a two-stage prediction process should be performed between 11 and 14 weeks, and between 19 and 24 gestational age, using MAP, UtA-PI and PIGF. Thanks to this modification, detection rates (DR) of $75 \%$ and $85 \%$ were achieved for the first and second trimesters, respectively; each with a false positive rate (FPR) of 10\% [15]. For the purposes of comparison, the model currently proposed by ACOG results in a DR of $90 \%$ with an FPR of $67 \%$ [16].

Later in the pregnancy, the highest predictive and diagnostic value starting from 20 weeks gestational age is achieved by measuring sFlt- 1 and PIGF levels simultaneously. The sFIt-1/PIGF ratio derived from this data is considered predictive at a cut-off point of 85 [17].

At the earliest, trophoblast invasion disorders lead to the development of clinical symptoms after 20 weeks gestational age. Research reported in the literature has shown that among patients with existing predictive factors for preeclampsia such as chronic hypertension, chronic renal conditions, diabetes mellitus andantiphospholipid syndrome, or with a history of preeclampsia, the sFlt-1/PIGF ratio test allows us to single out that group of patients who are at risk of developing a severe obstetric pathology at a given time. The test is similarly beneficial if conducted in pregnant patients who demonstrate any of the clinical or laboratory symptoms of preeclampsia [18]. Positive sFIt-1/PIGF ratio values, from tests repeated over a period at precise time intervals, allow us to dispel any diagnostic doubts that might persist in such situations.

2015 saw the presentation of the PROGNOSIS test that was carried out in 30 centers around the globe and that included 1.050 pregnant patients with suspected preeclampsia who were between 24- and 37-weeks gestational age. The study was designed to assess the usefulness of sFlt-1/PIGF ratio values for a short-term prognosis, of between 1 week and 4 weeks following the emergence of preeclampsia. Based on the study outcomes it was found that ratio values of less than 38 ruled out the emergence of preeclampsia within the following 7 days (an NPV of 99.3\%). The study showed that in patients below 34 weeks gestational age, with ratio values greater than 85 , and in those over 34 weeks gestational age, with ratio values greater than 110, there was a very high likelihood of them developing preeclampsia or another condition caused by an ischemic placenta.

In the high-risk cases mentioned above, the ratio values should be monitored and assessed for any indication of progression in the changes. In severe cases of early-onset preeclampsia with a ratio of $>655$, and in late-onset preeclampsia with a ratio of $>201$, there are strong implications for pregnancy termination within 48 hours of the test results. sFlt1/PIGF ratio values between 38 and 85 (110) indicate that 
there may be abnormal placental perfusion, therefore the authors suggest that in patients with these results prior to 34 weeks gestational age the ratio should be monitored, and in patients after 34 weeks gestational age the ratio should be monitored, and early labor induction considered [19].

Based on present studies, in 2016 the National Institute for Health and Care Excellence (NICE) of the United Kingdom found the sFlt-1/PIGF test to be a promising tool for ruling out preeclampsia in pregnant patients between 20- and 35-weeks gestational age. The Institute ruled, however, that there was a need for further study and that the current state of knowledge was insufficient for recommending the sFlt1/PIGF test as a routine tool in NHS practice [20].

In 2016 and 2017 more reports on the usefulness of the sFlt-1/PIGF ratio test for ruling out preeclampsia were published. Zeisler et al. presented their results of a multi-center study, where they proved that an sFlt/PIGF ratio cut-off level of 38 had a negative predictive value of $99.3 \%$ [21]. In another study of over 4.000 pregnant women with a low risk of developing preeclampsia, Sovio et al. [22] found that the sFIt-1/PIGF ratio was very useful for detecting preeclampsia after 36 weeks gestational age. In addition, the same study found that positive ratio values in women after 28 weeks gestational age correlated with a direct risk of the need to terminate the pregnancy due to evidence of clinical preeclamptic features. Dragan et al. presented the results of their research on the sFlt-1/PIGF ratio in more than 12,000 pregnant patients. The researchers showed that an sFlt-1/PIGF ratio of 38 or less provided a certainty of $99.97 \%$ that the patient would not deliver her child within a week of the test, due to preeclampsia. Moreover, the negative predictive value for a ratio of $>38$ was very high, at $99.91 \%$ [23].
As mentioned above, high sFlt-1/PIGF ratio values correlate positively with the manifestation of serious clinical symptoms necessitating pregnancy termination [18]. It has also been shown that higher sFlt-1 and lower PIGF concentrations are associated with the emergence of more severe clinical syndromes. In early-onset preeclampsia the ratio values are decidedly higher than in the late-onset form of this condition [24]. sFlt-1 concentrations are directly proportional to the severity of proteinuria and inversely proportional to the platelet count [25].

As the research carried out so far indicates, assessing angiogenesis disorder-related markers may prove to be a valuable support for and addition to the diagnosis of preeclampsia, and to the monitoring methods currently in use. The studies mentioned in our paper indicate clearly that the sFlt-1/PIGF ratio value also correlates with the severity of the clinical symptoms. Very high ratio values directly precede the emergence of symptoms necessitating pregnancy termination. Despite these facts, further studies are required before the sFlt-1/PIGF ratio can be introduced as an independent tool. This year has seen the publication of several reports that have included the sFlt-1/PIGF ratio in algorithms for predicting the development of early- and late-onset forms of preeclampsia. It has been proven possible to classify patients depending on their risk of preeclampsia already at 19-24 weeks gestational age [26], and then to compare the results at 30-34 [27] and at 35-37 [28] weeks gestational age. Historically, the highest sensitivity and specificity have been achieved by combining physical (MAP) and imaging (UtA-PI) examinations with biochemical (sFlt-1/PIGF) tests. The possible clinical applications of sFlt-1, PIGF and sFIt-1/PIGF ratio test results are shown in Figure 2.
11-14 and 19-24 weeks - across the population: PIGF, MAP, UtA PI

Prediction and classification in preeclampsia development

20-24, 30-34 and 35-37 weeks - across the population: sFlt-1, PIGF, MAP, UtA PI

Suspected preeclampsia

20-40 weeks - across

the population: sFlt-1, PIGF, MAP, Ut PI

Preeclampsia monitoring

Repeated assessment

of the sFIt-1/PIGFratio

Figure 2. Possible applications of sFIt-1, PIGF and the sFIt-1/PIGF ratio in prediction, diagnosis and monitoring of preeclampsia 
Our paper focuses on preeclampsia because most reports on the usefulness of the sFlt-1/PIGF ratio as a diagnostic tool have concentrated on this clinical syndrome. It must be emphasized, however, that the sFIt-1 and PIGF angiogenesis -related disease markers also demonstrate irregularities in other conditions related to placental hypoperfusion and ischemia. The markers show similar variation profiles in intrauterine growth restriction syndromes (IUGR) [29], and in some cases of placental abruption [30].

\section{SUMMARY}

Preeclampsia develops as a result of placental insufficiency. New angiogenesis markers sFlt-1 and PIGF provide an opportunity to predict, diagnose and monitor the status of placental angiogenesis abnormalities, including preeclampsia. According to present data, sFlt-1, PIGF and the sFIt-1/PIGF ratio permit the creation of a new model for preeclampsia management. The sFIt-1/PIGF ratio seems to be an especially valuable tool for use in clinical practice.

\section{REFERENCES}

1. Duley L. The global impact of pre-eclampsia and eclampsia. Semin Perinatol. 2009; 33(3): 130-137, doi: 10.1053/j.semperi.2009.02.010, indexed in Pubmed: 19464502.

2. Wallis AB, Saftlas AF, Hsia J, et al. Secular trends in the rates of preeclampsia, eclampsia, and gestational hypertension, United States, 1987-2004. Am J Hypertens. 2008; 21(5):521-526, doi: 10.1038/ajh.2008.20, indexed in Pubmed: 18437143.

3. Goldenberg RL, Rouse DJ. Prevention of premature birth. N Engl J Med. 1998; 339(5): 313-320, doi: 10.1056/NEJM199807303390506, indexed in Pubmed: 9682045.

4. Goldenberg RL, et al. Epidemiology and causes of preterm birth. Lancet. 2008; 371(9606): 75-84.

5. Ness RB, Sibai BM. Shared and disparate components of the pathophysiologies of fetal growth restriction and preeclampsia. Am J Obstet Gynecol. 2006; 195(1): 40-49, doi: 10.1016/j.ajog.2005.07.049, indexed in Pubmed: 16813742.

6. Orabona R, Donzelli CM, Falchetti M, et al. Placental histological patterns and uterine artery Doppler velocimetry in pregnancies complicated by early or late pre-eclampsia. Ultrasound Obstet Gynecol. 2016; 47(5): 580-585, doi: 10.1002/uog.15799, indexed in Pubmed: 26511592.

7. Bahado-Singh R, Syngelaki A, Mandal R, et al. Metabolomic determination of pathogenesis of late-onset preeclampsia. The Journal of Maternal-Fetal \& Neonatal Medicine. 2016; 30(6): 658-664, doi: 10.10 80/14767058.2016.1185411.

8. Romero R, Nien JK, Espinoza J, et al. A longitudinal study of angiogenic (placental growth factor) and anti-angiogenic (soluble endoglin and soluble vascular endothelial growth factor receptor-1) factors in normal pregnancy and patients destined to develop preeclampsia and deliver a small for gestational age neonate. J Matern Fetal Neonatal Med. 2008; 21(1): 9-23, doi: 10.1080/14767050701830480, indexed in Pubmed: 18175241.

9. Jardim LL, Rios DR, Perucci LO, et al. Is the imbalance between pro-angiogenic and anti-angiogenic factors associated with preeclampsia? Clin Chim Acta. 2015; 447: 34-38, doi: 10.1016/j.cca.2015.05.004, indexed in Pubmed: 25982781.

10. Eremina V, Sood M, Haigh J, et al. Glomerular-specific alterations of VEGF-A expression lead to distinct congenital and acquired renal diseases. J Clin Invest. 2003; 111(5): 707-716, doi: 10.1172/JCI17423, indexed in Pubmed: 12618525.

11. Wikström AK, Larsson A, Eriksson UJ, et al. Placental growth factor and soluble FMS-like tyrosine kinase-1 in early-onset and late-onset preeclampsia. Obstet Gynecol. 2007; 109(6): 1368-1374, doi: 10.1097/01. AOG.0000264552.85436.a1, indexed in Pubmed: 17540809.

12. Schrey-Petersen S, Stepan H. Anti-angiogenesis and Preeclampsia in 2016. Curr Hypertens Rep. 2017; 19(1): 6, doi: 10.1007/s11906-017-07065 , indexed in Pubmed: 28155021.
13. Chaiworapongsa T, Romero R, Kim YM, et al. Plasma soluble vascular endothelial growth factor receptor-1 concentration is elevated prior to the clinical diagnosis of pre-eclampsia. J Matern Fetal Neonatal Med. 2005; 17(1): 3-18, doi: 10.1080/14767050400028816, indexed in Pubmed: 15804781.

14. Leona CY. Poon, Nikos A. Kametas, NereaMaiz, Ranjit Akolekar and Kypros $\mathrm{H}$. Nicolaides. First-Trimester Prediction of Hypertensive Disorders in Pregnancy Hypertension. 2009; 53: 812-818.

15. Wright D, Gallo DM, Gil Pugliese $S$, et al. Contingent screening for preterm pre-eclampsia. Ultrasound Obstet Gynecol. 2016; 47(5): 554-559, doi: 10.1002/uog.15807, indexed in Pubmed: 26643929.

16. Gallo D, Wright $D$, Casanova $C$, et al. Competing risks model in screening for preeclampsia by maternal factors and biomarkers at 19-24 weeks' gestation. American Journal of Obstetrics and Gynecology. 2016; 214(5): 619.e1-619.e17, doi: 10.1016/j.ajog.2015.11.016.

17. Rana S, Powe CE, Salahuddin S, et al. Angiogenic factors and the risk of adverse outcomes in women with suspected preeclampsia. Circulation. 2012; 125(7): 911-919, doi: 10.1161/CIRCULATIONAHA.111.054361, indexed in Pubmed: 22261192.

18. Lapaire O, et al. , European Journal of Obstetrics \& Gynecology and Reproductive Biology. 2010; 151: 122-129.

19. Stepan Implementation of the sFIt-1/PIGF ratio for prediction and diagnosis of pre-eclampsia in signleton pregnancy: imlpication for clinical practic. UltrasoundObstetGynecol. 2015; 45: 241-246.

20. Vatish $M$, Strunz-McKendry $T$, Hund $M$, et al. sFlt-1/PIGF ratio test for pre-eclampsia: an economic assessment for the UK. Ultrasound Obstet Gynecol. 2016; 48(6): 765-771, doi: 10.1002/uog.15997, indexed in Pubmed: 27300726.

21. Zeisler H, Llurba E, Chantraine F, et al. Soluble fms-Like Tyrosine Kinase-1-to-Placental Growth Factor Ratio and Time to Delivery in Women With Suspected Preeclampsia. Obstet Gynecol. 2016; 128(2): 261-269, doi: 10.1097/AOG.0000000000001525, indexed in Pubmed: 27399996.

22. Sovio U, Gaccioli F, Cook E, et al. Prediction of Preeclampsia Using the Soluble fms-Like Tyrosine Kinase 1 to Placental Growth Factor Ratio: A Prospective Cohort Study of Unselected Nulliparous Women. Hypertension. 2017; 69(4): 731-738, doi: 10.1161/HYPERTENSIONAHA.116.08620, indexed in Pubmed: 28167687.

23. Dragan I, Wright D, Fiolna $M$, et al. Development of pre-eclampsia within 4 weeks of sFlt-1/PIGF ratio > 38: comparison of performance at 31-34 vs 35-37 weeks' gestation. Ultrasound Obstet Gynecol. 2017; 49(2): 209-212, doi: 10.1002/uog.17310, indexed in Pubmed: 27671743.

24. Romero R, Nien JK, Espinoza J, et al. A longitudinal study of angiogenic (placental growth factor) and anti-angiogenic (soluble endoglin and soluble vascular endothelial growth factor receptor-1) factors in normal pregnancy and patients destined to develop preeclampsia and deliver a small for gestational age neonate. J Matern Fetal Neonatal Med. 2008; 21(1): 9-23, doi: 10.1080/14767050701830480, indexed in Pubmed: 18175241.

25. Veas CJ, Aguilera VC, Muñoz IJ, et al. Fetal endothelium dysfunction is associated with circulating maternal levels of sE-selectin, sVCAM1, and sFlt-1 during pre-eclampsia. J Matern Fetal Neonatal Med. 2011; 24(11): 1371-1377, doi: 10.3109/14767058.2011.556204, indexed in Pubmed: 21381877.

26. Litwinska $M$, Wright $D$, Efeturk $T$, et al. Proposed clinical management of pregnancies after combined screening for preeclampsia at 19-24 weeks' gestation. UltrasoundObstetGynecol. ; 2017: Jan, doi: 10.1002/uog.17419.

27. Wright D, Dragan I, Syngelaki A, et al. Proposed clinical management of pregnancies after combined screening for pre-eclampsia at 30-34 weeks' gestation. Ultrasound Obstet Gynecol. 2017; 49(2): 194-200, doi: 10.1002/uog.17309, indexed in Pubmed: 27671544.

28. Panaitescu AM, Wright D, Militello A, et al. Proposed clinical management of pregnancies after combined screening for pre-eclampsia at 35-37 weeks' gestation. Ultrasound Obstet Gynecol. 2017; 50(3): 383-387, doi: 10.1002/uog.17419, indexed in Pubmed: 28133834.

29. Kwiatkowski S, Dołęgowska B, Kwiatkowska E, et al. A Common Profile of Disordered Angiogenic Factor Production and the Exacerbation of Inflammation in Early Preeclampsia, Late Preeclampsia, and Intrauterine Growth Restriction. PLoS One. 2016; 11(10): e0165060, doi: 10.1371/journal.pone.0165060, indexed in Pubmed: 27760191.

30. Signore C, Mills JL, Qian C, et al. Circulating angiogenic factors and placental abruption. Obstet Gynecol. 2006; 108(2): 338-344, doi: 10.1097/01.AOG.0000216014.72503.09, indexed in Pubmed: 16880304 